\title{
LO SPASIMO DI PALERMO FRA CRISI E MEMORIA
}

\author{
Cinzia Gallo \\ Università di Catania \\ cinzia.gallo@tin.it \\ cinziagll@virgilio.it
}

\section{Riassunto}

Lo Spasimo di Palermo è esempio di una crisi che ha ripercussioni nella forma espressiva: da una parte, emerge la consapevolezza che «sul ciglio dell'abisso la parola si raggela, si fa [...] simbolo sfuggente», dall'altra il romanzo appare un "genere scaduto, corrotto, impraticabile». Il conflitto generazionale fra Chino e Mauro rientra pure in quest'ambito. Da qui derivano varie soluzioni che portano al "poema narrativo» di Consolo e testimoniano il disorientamento prevalente: accumuli paratattici, metafore, figure retoriche, figure del discorso. Le suggestioni pittoriche trovano il loro centro nello Spasimo di Sicilia di Raffaello, simbolo di una sofferenza che da Palermo arriva alla Sicilia e a tutto il mondo, mentre gli spazi hanno una grande importanza e mostrano la perdita della memoria storica, responsabile della crisi.

Parole chiave: Consolo, crisi, memoria, storia

Secondo Ugo Dotti, Lo Spasimo di Palermo è caratterizzato dal «contrapporsi di storia a memoria, nel vano bisogno di conforto che il 
ricordo potrebbe dare e che suscita al contrario un cocente sentimento di patimento, di sconfitta, di spasimo» (Dotti, 2012, 315). Dunque, uno stretto nesso fra storia, crisi e memoria percorre l'intero testo, anche se esso è, innanzitutto, specchio di una crisi, sia collettiva che individuale. Consolo presenta non solo «il fallimento della generazione del dopoguerra nel creare una società più giusta e più civile» (O'Connell, 2008, 173), per cui terrorismo e mafia risultano uniti «in un clima da fine dei tempi, che nega la storia» (Donnarumma, 2011, 446), ma anche la sconfitta di Chino, padre e scrittore. Da ciò l'impossibilità di narrare, cioè di utilizzare i tradizionali mezzi espressivi. Raccontare è però necessario, come attestano le due battute del Prometeo incatenato di Eschilo poste in epigrafe: «Rivela tutto, grida il tuo racconto... / Il racconto è dolore, ma anche il silenzio è dolore» (Consolo, 1998 , 7). Quest'urgenza di dire si realizza subito tramite la letteratura, come nella conclusione di Nottetempo, casa per casa, in cui essa appare «ultima consolazione $[\ldots]$ e ultima possibilità di spiegazione del "dolore" del mondo» (Luperini, 1999, 166). Lo stesso Consolo chiarisce: «[...] è caduta la fiducia nella comunicazione, nella possibilità [...] della funzione sociale, politica della scrittura. Non rimane [...] che l'urlo o il pianto, o l'unica forza oppositiva alla dura e sorda notte, la forza della poesia» (Consolo, 1993, 58). Il gioco citazionistico è, quindi, subito in primo piano: l'iniziale «Allora tu, [...] ed io» (Consolo, 1998, 9), con cui il narratore si rivolge a Chino, è stato collegato da O'Connell $(2008,181)$ al celebre verso di Eliot «Let us go then - you and I», con cui, peraltro, si chiude il quinto capitolo. Un'altra citazione eliotiana («In my beginning is my end» [Consolo, 1998, 9]), rimandando al consueto simbolo consoliano della chiocciola, della spirale, rileva invece l'importanza della memoria, necessaria per recuperare un'identità veicolata dai nomi («il nome tuo d'un tempo, il punto di partenza» [Consolo, 1998, 9]), di cui Consolo sottolinea spesso l'importanza come segno identitario.

Queste citazioni forniscono subito una prima chiave di lettura, in quanto Eliot utilizza termini spesso non uniti fra loro da legami logici, alterna espressioni auliche con altre colloquiali, mescola svariate lingue, rappresentando così la crisi esistenziale dell'uomo: similmente si comporta Consolo. Anche nel nostro testo, comunque, il narrare significa «rappresentare il mondo, cioè ricrearne un altro sulla carta» (Consolo, 2012, 92), che «assolva la tua pena, il tuo smarrimento» (Consolo, 1998, 
10). Questi stati d’animo, già nel primo capitolo, sono rapportati da Chino, giunto a Parigi per incontrare il figlio, con la parola che «si fa suono fermo, [...] simbolo sfuggente» (Consolo, 1998, 12). Egli, dunque, respinge come false la lingua accademica («Stendono prose piane i professori, [...] decorano le accademiche palandre di placche luccicanti» [Consolo, 1998, 12]) e quella giornalistica ( «Nul néchappe décidément, au journalisme ou voudrait-il...» [Consolo, 1998, 12]), e nega il potere evocativo del linguaggio: se dapprima, infatti, il prete oppone alla forza delle armi quella delle parole (Giacomo - Marcellesi, 2004, 73-74), quando chiede, allinizio dei bombardamenti, «di cantare l'aria con parole senza senso» (Consolo, 1998, 14), queste, poi, non servono a disincantare una «trovatura» (Consolo, 1998, 19). Tornano in mente a Chino, invece, termini legati ad epoche passate («Il maggiore parlò in un modo che credeva ancora di quel luogo» [Consolo, 1998, 13]). Per Consolo, difatti, «la vera scrittura [...] poggia sulla memoria letteraria soprattutto» (Consolo, 2006, 71 - 72). O’Connell l'ha definita «un ricco mosaico di intertestualità, le cui tessere sono fatte di testi sia antichi sia moderni», in quanto pone accanto «linguaggi e dialetti diversi e spesso stridenti tra di loro», oscillando «tra registro alto e basso» (O’Connell, 2008, 164). Nei primi due capitoli, alcuni termini dialettali o colloquiali («anciove» [Consolo, 1998,15]; «caruso», «scarparo» [Consolo, 1998, 26]; «buatta» [Consolo, 1998, 32]; «a forma di lasagne» [Consolo, $1998,25])$ coesistono, dunque, con altri più elevati, o desueti, formando uno strano impasto linguistico, espressione dello smarrimento in cui vive Chino nel tentativo di superare i fantasmi del passato. Lo stesso Consolo ha dichiarato, in un'intervista: «sono stato $[. .$.$] sperimentatore, senza però$ arrivare allestensione linguistica e alla straordinaria polifonia gaddiana, ma operando verticalmente, nel senso di riportare in superficie, [...] parole e strutture di lingue sepolte» (Ciccarelli, 2005, 96). Si inserisce in tale ambito il termine "marabutto», di origine araba e perciò legato alla storia della Sicilia, che ritornando varie volte nel nostro testo, ne suggellerà landamento circolare svelando il suo carattere di «metafora malinconica» (Traina, 2001, 107). Rientra in questa circolarità anche il rilievo dato alle immagini, in quanto Consolo avverte «Sempre [...] l'esigenza di equilibrare la seduzione $[\ldots]$ della parola con la visualità, con [...] una concretezza visiva» (O’Connell, 2004, 241). 
Il primo capitolo, così, accosta, secondo una tecnica cinematografica, parti quasi autonome, corrispondenti alle vicende affiorate alla memoria di Chino. Non a caso l'albergo di Parigi in cui egli soggiorna, oltre a chiamarsi La dixième muse, mostra alle pareti foto di vari divi del cinema, e alla mente di Chino ritorna l'immagine del IUDEX, protagonista del serial cinematografico di Feuillade che adesso egli vede per intero, dopo cinquant'anni, e che sarà richiamato anche alla fine del libro. L'ultimo segmento del capitolo presenta invece il dolore di Chino quale dolore collettivo attraverso il tipico uso consoliano delle figure retoriche, confermando l'orientamento dello scrittore verso il ben noto poema narrativo:

Lo strazio fu di tutti, di tutti [anadiplosi] nel tempo il silenzio fermo [allitterazione], la dura pena, il rimorso scuro [chiasmo], come d'ognuno ch'è ragione, [...] d'un fatale arresto, d'ognuno [anafora] che qui resta, o di qua d'un muro, d'una grata, parete di fenolo [metafora], vacuo d'una mente, davanti alla scia in mare, all'arco in cielo che dispare di cherosene [iperbato]. L'esilio è nella perdita, l'assenza, in noi l'oblio, la cieca indifferenza [omoteleuto] (Consolo, 1998, 24).

Un'anastrofe («il tempo suo più avventuroso» [Consolo, 1998, 25]) conclude invece le enumerazioni con cui si esprime lo stato di abbandono di Chino dopo la morte del padre. Analogamente, le enumerazioni indicano il disordine, il caos che contraddistinguono il viaggio di Chino ed Urelia, il loro arrivo a Palermo:

Il viaggio fu infinito, nel fumo, nel freddo, raffiche di vento, pioggia, spruzzi delle onde, stretti fra reduci, sbandati, intrallazzisti, donne scasate e di mestiere, ragazzi fuggitivi, frati di questua e ceffi di galera. [...]

Mai erano stati Urelia e Chino nella città, mai avevano udito tanto chiasso, urla richiami imbonimenti, visto tanto correre e affannarsi tra macerie, travi fili ferri lamiere tufi calcinacci, cantoni in bilico, tappezzerie e maioliche esposte all'aria, giare e bidoni su lastrici crollati, statue riverse, guglie mozzate e cupole sventrate (Consolo, 1998, 31). 
Spiccano l'aggettivo dialettale «scasate», il neologismo «intrallazzisti» tra termini più elevati, quali «questua», «fuggitivi», mentre gli aggettivi «riverse», «mozzate», «sventrate» umanizzano gli oggetti, rendendo più forte la sensazione di sofferenza. A Palermo, i «ciechi vaiolosi, storpi, appestati d'ogni sorta che dallo Spasimo si recavano ogni giorno per mangiare alla Dogana» (Consolo, 1998, 32) ricordano l' «orda di mendichi, ciechi, storpi, nani, malformati» che, in Retablo, si presentano davanti a Clerici, «con lamenti, cantilene e preghiere strazianti» (Consolo, 1992, 51). Con queste autocitazioni, Lo Spasimo di Palermo si conferma, come la critica unanime ha riconosciuto, testo che riassume l'evoluzione artistica ed ideologica di Consolo.

Dopo un'ellissi, intanto, tornati nel tempo primo della narrazione, il terzo capitolo inizia, significativamente, con la parola «orrore» (Consolo, 1998, 33): è lo stato d'animo di Chino davanti al suo volto ritratto nello specchio, segno, perciò, quasi, di un'intima dissociazione, che egli descrive, grazie alle enumerazioni, espressionisticamente. Del resto, Norma Bouchard ha riscontrato, ne Lo Spasimo, «[...] an expressionism so radical that it has baffled more than one critic» (Bouchard, 2005, 6). Il pensiero subito rivolto al padre testimonia la persistenza di una lacerante distanza, messa in risalto dall'interrogativa: «Fosse vissuto, sarebbe scaduto così anche suo padre, avrebbe avuto quella faccia, quella sagoma avvilita, si sarebbe piegata la sua testa, il suo orgoglio si sarebbe [chiasmo] con gli anni incenerito [metafora]?» (Consolo, 1998, 33). La stessa incomunicabilità impernia, adesso, il suo rapporto con il figlio Mauro: le parole appaiono infatti «una pazzia recitata, un teatro dell'inganno» (Consolo, 1998, 35) quando i due si incontrano, in un clima di precarietà evidenziato da metafore: «Chi può sapere in questo mulinello di sagome simili e cangianti, questo turbinio di figure, quest'infinito scorrer d'apparenze?» (Consolo, 1998, 34). Se, da una parte, tale incertezza si manifesta, nuovamente, a livello individuale, per cui Chino è consapevole di non essere «riuscito a placare» i suoi «assilli» (Consolo, 1998, 36), «il panico, l'arresto, [...] l'impotenza, l'afasia, il disastro» della sua vita, dall'altra lo «scadimento» (Consolo, 1998 , 37) è generale, rappresentato dai gusti letterari dei frequentatori della libreria che Daniela ha voluto, per tentare di diffondere romanzi e poesie fra i compagni, «ignari e sprezzanti d'ogni forma letteraria» (Consolo, 
1998, 37). Responsabile è l'omologazione, lo smarrimento della memoria storica, il predominio delle «mode» (Consolo, 1998, 37): nella vetrina della libreria, antifrasticamente chiamata «La porta d'Ishtar», notiamo allora «la foto d'un giovanotto levigato, riccioletti e occhio vellutato [omoteleuto], ultimo autore di successo, mistico e divo della tivù, [...]» (Consolo, 1998, 36). Rafforza questo svilimento della memoria culturale il menu del ristorante Les philosophes: «Cotolette Spinoza - Salade Bergson - Salade Platon - Salade Aristote - Coupe Virgile - Coupe Socrate» (Consolo, 1998, 38). Mauro, poi, che enumera i mali italiani («Sempre nel marasma, nel fascismo inveterato, nell'ingerenza del pretame, nella mafia statuale il paese beneamato?» [Consolo, 1998, 39]), sottolineando le responsabilità del fascismo con l'omoteleuto («inveterato [...] beneamato») e l'allitterazione («marasma [...] fascismo»), rivolge al padre delle accuse ben precise, rese più incisive dalle metafore, dall'omoteleuto (ornamento-annientamento), dall'anafora, e che coinvolgono tutti i letterati: «tu e i soavi letterati siete le epigrafi d'ornamento, la lapide incongrua e compiaciuta sul muro di quel carcere mentale, quel manicomio d'annientamento» (Consolo, 1998, 39). I riferimenti al romanzo El recurso del método di Carpentier, autore particolarmente caro a Consolo, alla vicenda di Camille Rodin ribadiscono, poi, ancora, la volontà di distacco da una realtà costituita da un «tempo feroce e allucinato» (Consolo, 1998, 42).

Il capitolo IV si apre allora con un lungo periodo che accosta immagini metaforiche apparentemente distinte, in realtà accomunate da un senso di negatività, dolore, precarietà, sottolineato dalle enumerazioni miste ad anafore e assonanze:

Muro che crolla, interno che si mostra, fuga affannosa, segugio che non molla [assonanza], esito fra ruderi sferzati dalla pioggia, ironiche statue in prospettiva, teschi sui capitelli, maschere sui bordi delle fosse, botteghe incenerite, volumi che in mano si dissolvono, lei al centro d'un quadrivio accovacciata, lei distesa nella stanza che urla e che singhiozza, ritorna dall'estrema soglia, dall' insulinico terrore, entra ed esce per la porta sull'abisso, il tempo è fisso nel continuo passaggio, nell'assenza, nel fondo sono le sequenze, i nessi saldi e veri (Consolo, 1998, 45). 
La serie di metafore può apparentarsi al flusso di coscienza di Joyce, il cui Ulisse Consolo definisce «L'odissea dei vinti» ${ }^{[1]}$ (Consolo, 1999, 111). D’altra parte, vedere il film della sua giovinezza non risolve i problemi di Chino ma gli fa capire l'importanza dell'immaginazione e della memoria ${ }^{[2]}$ : «Il tempo, la memoria esalta, abbellisce ogni pochezza, ogni squallore, la realtà più vera. Per la memoria, la poesia, l'umanità si è trasfigurata, è salita sull'Olimpo della bellezza e del valore» (Consolo, 1998, 53). La reazione di Mauro («"Ne hanno combinate i letterati!”» [Consolo, 1998, 53]) conferma che il contrasto generazionale con il padre coinvolge pure la funzione della letteratura e dei letterati, come si chiarirà nella conclusione. L'incomunicabilità fra i due sembra venir meno solo quando Chino dice al padre: «Portale [alla madre, Lucia] per me, quando sarà fiorito, il gelsomino» (Consolo, 1998, 55), mostrando così che, pure per lui, i legami con la Sicilia, di cui quel fiore è simbolo, al pari di quello che avviene ne L'olivo e l'olivastro, sono ineludibili. Per Chino, invece, ritornare in Sicilia significa ripercorrere le vicende della propria vita, che somiglia sempre più ad una fuga, per placare le proprie angosce. Milano e la Sicilia, ovvero $\mathrm{i}$ «due poli» entro cui si svolge la vicenda di questo e di molti testi consoliani, si precisano quali simboli «di una condizione di esilio perenne» (Lollini, 2005, 32). Da qui le tantissime «metafore dell'esilio, dell'erranza» (Lollini, 2005, 29), la ricorrenza dei termini 'esilio-esule', 'fuga-fuggire, 'partire-partenza', 'evadere-scappare', insieme a quelli relativi al campo semantico del dolore.

Il quinto capitolo registra questi concetti in modo particolare. Il primo segmento esprime ancora il disagio di Chino con i procedimenti già notati: brevi proposizioni cooordinate per asindeto, enumerazioni. Spicca il chiasmo «lame squame gemini binari» (Consolo, 1998, 57), in cui i due aggettivi aulici ('squame', antico; 'gemini', latinismo) sono incastonati fra i sostantivi 'lame, binari', del registro quotidiano. Segue un'analessi in cui vita e letteratura appaiono strettamente unite, visto che i libri hanno scandito le varie tappe dell'esistenza di Chino. Inverano, intanto, lo spazio: Chino si reca con Lucia, infatti, a visitare la tonnara e il mare descritti nelle

[1] Un'allusione all'Ulysses di Joyce e «the re-inauguration of the modern nostos into twentieth-century literary culture», si troverebbe, secondo O'Connell $(2012,246)$, nel seguente passo: "Ascesero man mano e si dispersero per i vari cieli, entro le celle di quella Sandycove dell'introibo, teca babelica, averno del viaggio» (Consolo, 1998, 46).

[2] Secondo O'Connell (Consolo narratore cit., 178), si ha qui un richiamo al saggio Angelus Novus di Benjamin, che peraltro Consolo cita in I ritorni, ed esattamente ai concetti di «rimembranza» e memoria. 
Osservazioni pratiche intorno la pesca, corso e cammino dei tonni. Quando i poliziotti perquisiscono la sua casa, come si narra anche in Un giorno come gli altri, i suoi libri sparpagliati sembrano «storie perenti, lasche prosodie, tentativi inceneriti, miseri testi della sua illusione, del suo fallimento» (Consolo, 1998, 66), dovuto, comunque, al «tempo feroce, disumano» (Consolo, 1998, 67) in cui vive. Lo testimoniano i procedimenti espressivi. Il «boemo paonazzo», in cui si riconosce Milan Kundera, definisce infatti «merde» gli intellettuali insensibili ai problemi della primavera di Praga nella discussione tra le signore che, con allusione ad Eliot, «in seriche casacche orientali andavano e venivano, [...] rivolgevano domande a Saul Bellow» (Consolo, 1998, 66). Il narratore, poi, inserisce la parola antica 'grascia' in un'enumerazione («ingombro, grascia, fermento, trionfo di laidume, baccano d'osceno carnevale» [Consolo, 1998, 68]) per raffigurare la disastrosa situazione di Milano.

Essa, la «città ignota» è poi descritta con una citazione da Excelsior di Luigi Manzotti («"D’improvviso la scena si trasforma, la Luce e la Civiltà trovansi abbracciate..."» [Consolo, 1998, 69]), menzionato pure in Sopra il vulcano, e una dai Promessi Sposi ("Quel contrapposto di gale e di cenci, di superfluità e di miseria”» [Consolo, 1998, 70]). Il segmento successivo rielabora il brano I barboni, scritto nel 1995 per il catalogo di Ottavio Sgubin, che termina con una citazione dell'Odissea, l'unica di tutto Lo Spasimo ("Di' perché piangi e nel tuo animo gemi / quando odi la sorte...» [Consolo, 1998, 72]), che, non a caso, rimanda ad una condizione di sofferenza. Lo stesso Consolo ne chiarisce, ne Lo spazio in letteratura, il significato, collegando il nostro testo con L'olivo e l'olivastro. Quindi risponde alla richiesta contenuta nella citazione dell'Odissea sostenendo l'impossibilità della narrazione: «"Io sono... no, no, l'aridità, la lingua spessa, l'oblio d'ogni nesso... illuso ancora dell'ascolto, tu procedi"» (Consolo, 1998, 72), affermazione apparentemente in contrasto con il montaliano «varco» che avrebbe condotto Chino, all'inizio del capitolo, «nel passato, nel racconto, in cui [...] tutto sembrava decifrabile» (Consolo, 1998, 69). In effetti, nell'analessi in cui il narratore rievoca la vita familiare inizialmente felice di Chino, di Lucia e poi devastata dalle circostanze, la letteratura è dapprima celebrata come depositaria della «verità umana» (“"Là si trova” [...] "negli assoluti libri, la verità umana"») ma poi, di fronte 
al precipitare degli eventi, ritenuta inadeguata a riprodurre correttamente la realtà («Ogni parola ora, povera, incapace, riduce quell’incanto, [...]» [Consolo, 1998, 73]). Consolo, allora, da un canto adotta una tecnica cinematografica, mostrando i vari eventi (le intimidazioni, la conversazione con il commissario, la vendita della casa) come fotogrammi, dall'altro ritorna alla 'narrazione poematica' propria de L'olivo e l'olivastro ${ }^{[3]}$, a confermare la corrispondenza tra i due testi. Così è facile riconoscere degli endecasillabi quando il narratore riferisce la decisione di Chino di lasciare Palermo per andare sulle tracce dell'originaria ed autentica identità siciliana. E il viaggio, vero e proprio topos per Consolo, avviene, significativamente, durante la Pasqua, momento di resurrezione: «S'era fatta smunta, pallida, inquieta /, [...] Chino decise di rompere l'assedio, / d'evadere, fuggire dalla casa, / [...] Andò con l'infelice per le strade, / [...]. Andò in un aprile, nel tempo della Pasqua [due settenari]» (Consolo, 1998, 76-77). Una serie di immagini, combinate con un'anafora e un endecasillabo finale, presenta la Sicilia quale metafora del mondo: «Era là il centro dello spazio, la visione sconfinata, là il cuore della terra, / del mito più oscuro e luminoso» (Consolo, 1998, 77). Il nome di Borges, del resto, rimanda non solo ai «sogni», agli «incubi», agli «specchi», ai «labirinti» (Consolo, 1998, 80), temi presenti nel nostro testo, ma al suo prevalente carattere metaforico. La vicenda di Lucia, con il suo «precipitare nel gorgo medicale, nell'ignoranza, nel dominio, nel cinico interesse di luminari, case di cura, [...] rete di sciacalli» (Consolo, 1998, 78) e quella di Chino testimoniano i danni causati dalla perdita della memoria storica: Palermo è, difatti, ormai, una città «stravolta, squallida nell'uniforme volto, nell'anonima sua morsa, nel cieco manto sopra ogni verde luce, nella grigia muraglia avanti a vecchi squarci, immobili macerie, [...]» (Consolo, 1998, 79).

All'insegna della metafora si svolge, allora, il capitolo settimo. «un re che narra e che governa, elude la metafora, annulla la contraddizione della prosa» (Consolo, 1998, 83) è, intanto, il personaggio principale del racconto letto dal glottologo protagonista de La perquisizione, dietro cui la critica ha scorto Un giorno come gli altri. La metafora si precisa, dunque, quale una necessità, legata a una narrazione in crisi, che tratta di periodi in crisi: appaiono così chiari i rapporti di Consolo con il postmodernismo. $\mathrm{Da}$

[3] Esso è l'«apice» di una vera e propria «sperimentazione "poetica"» (Francese, 2015, 70) 
una parte vi sembrerebbero rimandare il citazionismo, il plurilinguismo, il tema del complotto; dall'altra, però, Consolo se ne mantiene distante, proprio per l'importanza attribuita alla memoria e alla storia, come Norma Bouchard ha puntualizzato: «it is important to point out that even though Consolo's novels exhibit many of the rhetorical devices that we have come to associate with postmodern writing practices, they also remain fundamentally distinct from dominant, majoritarian forms of postmodernism» (Bouchard, 2005, 10-11).

Una «metafora perenne» giudica poi Chino il romanzo di Manzoni, modello per Consolo di romanzo storico, in quanto allude «alle pesti in ogni tempo di Milano» (Consolo, 1998, 85). Il «lazzaretto più appestato» sembra a Chino «una piazzetta» in cui, avvolti in un'omologazione che cancella ogni identità, «Stavano ragazzi barcollanti o immobili, il busto avanti, piegati sui ginocchi. Sembravano bloccati in quelle pose, pietrificati [...]» (Consolo, 1998, 85). Modellato sull'Addio ai monti di Manzoni è, inoltre, l'Addio che Consolo rivolge a Milano, «Città perduta» (Consolo, 1998, 91) non meno della Milano di Berlusconi e della Lega Nord, condannata attraverso la consueta tecnica dell'accumulo:

\begin{abstract}
Illusione infranta, amara realtà, scacco pubblico e privato, castello rovinato, sommerso dall'acque infette, dalla melma dell'olona, dei navigli, [...] scala del corrotto melodramma, palazzo della vergogna, duomo del profitto, basilica del fanatismo e dell'intolleranza, banca dell'avventura e dell'assassinio, fiera della sartoria mortuaria, teatro della calligrafia, stadio della merce e del messaggio, video dell'idiozia e della volgarità (Consolo, 1998, 91).
\end{abstract}

Così, quando Mauro chiede al padre se stia scrivendo, egli risponde negativamente. E se il suo comportamento è differente da quello di altri scrittori (Calvino, Moravia, Sciascia, indicati con delle metafore - «il castoro ligure, il romano indifferente, l'amaro tuo amico siciliano»), ciò accade perché essi hanno «la forza [...] della ragione, [...] la geometria civile dei francesi» (Consolo, 1998, 88): non vivono cioè una condizione di disorientamento. Costituiscono dunque, queste parole, opinioni dello stesso Consolo (Chino Martinez sarebbe cioè un alter ego dello scrittore), che, proseguendo il suo discorso, una sorta di dichiarazione di poetica, 
confessa: «mi perdo nel ristagno dell'affetto, l'opacità del lessico, la vanità del suono...» (Consolo, 1998, 88). La letteratura, però, deve essere, soprattutto, espressione di una società, tant'è che Mauro, in carcere, chiede al padre di portargli I demoni di Dostoevskij, L'affaire Moro di Sciascia, Scritti corsari di Pasolini, Une saison en enfer, Illuminations di Rimbaud.

Chino, allora, abbina alla letteratura, che perciò rappresenterebbe un'ancora di salvezza come nella conclusione di Nottetempo (si rifugia nella biblioteca, per esempio), il valore della memoria, delle radici, individuali e collettive. Sul treno per Napoli, infatti, ascoltando parlare dei ragazzi, individua facilmente, e con piacere, «le città e i paesi da cui quei ragazzi provenivano. [...] Leggeva in quel concerto la storia d'ogni luogo, i segni [...] superstiti delle migrazioni, dei remoti insediamenti» (Consolo, 1998, 95). Egli, pertanto, considera negativamente, al punto da definirla «trucida» (Consolo, 1998, 94), la nuova lingua annunciata da Pasolini, in quanto segno di un'omologazione negatrice della storia. Di questa sono testimonianza i luoghi, lo spazio, che Consolo ritiene, sempre, molto importanti. In prossimità di Palermo Chino scorge, difatti, «il piano di Sant'Erasmo, la foce melmosa dell'Oreto, [...] la Porta dei Greci, [...] gli antichi palazzi dietro nobiliari, le cupole e i campanili delle chiese, il Càssaro Morto e la Porta Felice, Santa Maria della Catena, la conca stagna affollata d'alberi di lussuose barche della Cala» ma anche «le palazzate nuove del sacco mafioso» (Consolo, 1998, 98). Attraverso lo spazio, cioè, è possibile leggere i cambiamenti della società. Se i luoghi, allora, come la letteratura, manifestano la memoria storica, Chino sente di dover «ricominciare» dai libri, oltre che «dalla chiara geografia» (Consolo, 1998, 102). Si presentano, questi, strettamente uniti, interdipendenti nelle pagine finali dello Spasimo, che, perciò, chiarisce e conclude l'esperienza letteraria di Consolo, rappresentando «al contempo la mediazione verso» quella che Consolo considera una vera e propria «impasse e la risposta ad essa» (O'Connell, 2008, 174). Così, dopo aver raccolto notizie sul musicista D'Astorga, da cui prende nome la strada in cui abita, ed aver scoperto il quartiere intorno, aver collegato il castello della Favara ad alcuni versi del poeta arabo Ab dar-Rahmân, Chino decide di «indagare sulla prigionia in Algeri di Cervantes», su quella di Antonio Veneziano ${ }^{[4]}$ e di scrivere «della

[4] Ne L'olivo e l'olivastro (1994, 105), Consolo afferma che, ad Algeri, Cervantes scrive dei versi per Antonio Veneziano. 
pena vera di due poeti, fuori da ogni invenzione» (Consolo, 1998, 105). Infatti,

Aborriva il romanzo, questo genere scaduto, corrotto, impraticabile. Se mai ne aveva scritti, erano i suoi in una diversa lingua, dissonante, in una furia verbale ch'era finita in urlo, s'era dissolta nel silenzio. Si doleva di non avere il dono della poesia, la sua libertà, la sua purezza, la sua distanza dall'implacabile logica del mondo (Consolo, 1998, 105).

Consolo ripropone, in tal modo, le riserve nei confronti del romanzo manifestate in altri testi (Fuga dall' Etna, Nottetempo, L'olivo e l'olivastro).

Un «libro raro» (Consolo, 1998, 106), in spagnolo, trovato in biblioteca, conferma le sue convinzioni. Vi legge di ogni regione d'Italia e del mondo, apprende i vari significati, positivi, del termine 'marabutto'. È consapevole, anche per questo, di come i tragici eventi che avevano portato alla morte del padre avessero costituito una stortura. Poi, dopo aver vagato per le strade di Palermo ed aver visto, nella «casba di Seralcadio» (Consolo, 1998, 108), un'altra faccia della città, come avvenuto a Milano, si ferma nella chiesa di Santa Maruzza, luogo carico di ricordi, personali e letterari, opposto al drammatico presente. Difatti, «Dentro era l'accesso, racconta il Natoli, alle caverne, alle camere segrete dei processi e delle sentenze dei giustizieri» (Consolo, 1998, 108). Ma al tribunale dei Beati Paoli si lega il vero Palazzo di Giustizia, a cui Chino giunge seguendo un «corteo, fantasmatico» (Consolo, 1998, 109). Il suo pensiero va, allora, al lavoro dei magistrati contro i gruppi mafiosi e, in particolare, al «figlio della sua dirimpettaia» (Consolo, 1998, 109), cioè il giudice Borsellino. L'incontro fra i due avviene nel segno dei libri e conferma come Chino sia alter ego di Consolo «Ho letto i suoi libri... difficili, dicono» (Consolo, 1998, 115), dice il giudice che, dopo aver citato un passo de Le pietre di Pantalica («Palermo è fetida, infetta. In questo luglio fervido esala odore dolciastro di sangue e gelsomino...» [Consolo, 2012, 132]), commenta: «Ma nulla è cambiato, creda. Vedrà, il prossimo luglio sarà uguale... o forse peggio» (Consolo, 1998, 115). Egli legittima, in tal modo, la funzione civile della letteratura, legata anche ad altre arti. Chino trova, infatti, nella «memoria 
d'un anonimo spagnolo», delle notizie sulla tela che Raffaello dipinge per la chiesa di Santa Maria dello Spasimo, e che intitola «sgomento della Vergine e Spasimo del Mondo» (Consolo, 1998, 112). Giustamente, dunque, la Sicilia è metafora del mondo: la sua crisi, la sua degenerazione sono generali, così come "questo Spasimo, da Palermo» (Consolo, 1998, 112) coinvolge la Sicilia e il mondo tutto. E i luoghi, come la letteratura testimonia, lo confermano:

Lesse di Santa Maria dello Spasimo abbandonata dai frati per il nuovo baluardo di difesa che a ridosso il viceré don Ferrante Gonzaga fece costruire. Della magnifica chiesa che divenne poi nel tempo teatro, lazzaretto nella peste, granaio, magazzino, albergo di poveri, sifilicomio, cronicario, luogo di dolore, solitudine, abbandono (Consolo, 1998, 113).

Una metafora indica il perdurare di questa condizione, ormai generalizzata, al presente: lo scirocco è un «sudario molle» (Consolo, 1998, 113). Le immagini mettono poi, in parallelo, la peste e il colera, che nel passato hanno oppresso Palermo, con la mafia. $\mathrm{Al}$ «paranzello o brigantino» che arrivava a Palermo «con l'infetto» (Consolo, 1998, 113) corrisponde «il corpo sfatto, quasi scheletrico» (Consolo, 1998, 116) rinvenuto dentro il pilastro del portico del palazzo dove abita Chino, il quale ricorda i vv. 10-12 del quarto canto dell'Inferno quale metafora dell'incapacità di trovare una soluzione a questa situazione: «Oscura e profonda era e nebulosa / tanto che, per ficcar lo viso a fondo, / io non vi discernea alcuna cosa» (Consolo, 1998, 118). L'ultimo capitolo si svolge dunque la domenica successiva al Festino di Santa Rosalia, per sottolineare come pure la Santa che aveva salvato Palermo dalla peste, nel 1624, sia adesso impotente. La conformazione della città, con «il fitto ammasso dei palazzi, il cantiere dietro il muro, la corta via d'Astorga [allitterazione]», mostra la perdita di ogni memoria storica, «sepolta sotto il cemento» (Consolo, 1998, 123). Le enumerazioni, mettendo insieme termini di origine araba ('càssaro', 'kalsa'), spagnola ('criadi'), neologismi ('villena'), rappresentano questa condizione di disordine, che la metafora conclusiva suggella: «Congiura, contagio e peste in ogni tempo» (Consolo, 1998, 123). 
Alla memoria storica, invece, ci riporta il fioraio che in «questa città infernale» (Consolo, 1998, 126), in cui ogni ragionevolezza sembra essere venuta meno, si chiama, non a caso (il riferimento è ad Erasmo da Rotterdam e al suo Elogio della follia), Erasmo, e che, dopo aver donato a Chino dei gelsomini, gli rivolge delle parole, apparentemente oscure, inquietanti, ma che legano il suo passato al suo presente: «Ddiu ti scanza d'amici e nnimici, e di chiddi / chi ti manciunu lu pani. / Ddiu ti scanza di marabutta» (Consolo, 1998, 124). Ciò è evidente nella lettera che Chino scrive al figlio, in cui troviamo insieme i cinque romanzi che Massimo Onofri ha individuato fra le pagine de Lo Spasimo: «un romanzo sul rapporto Italia - Sicilia; un romanzo sul rapporto padre e figlio (il padre di Gioacchino / Chino e Chino); un secondo romanzo padre - figlio (Chino e Mauro); [...] un romanzo d'amore (Chino e Lucia); e [...] un romanzo di "oblio e dimenticanza"» (Onofri, 2004, 183). Si precisano qui, quando Chino allude all'omicidio del giudice Falcone, le corrispondenze con il passo de Le pietre di Pantalica citato da Borsellino: «Questa città, lo sai, è diventata un campo di battaglia, un macello quotidiano. Sparano, fanno esplodere tritolo, straziano vite umane, carbonizzano corpi, spiaccicano membra su alberi e asfalto - ah l'infernale cratere sulla strada per l'aeroporto! - E' una furia bestiale, uno sterminio» ${ }^{[5]}$ (Consolo, 1998, 128). Ne Le pietre di Pantalica aveva detto: "Questa città è un macello, le strade sono carnazzerie con pozzanghere, rivoli di sangue coperti da giornali e lenzuola. [...] La guerra contro la civiltà, la cultura, la decenza» (Consolo, 2012, 132 - 134). E, ne L'olivo e l'olivastro, si indica in modo chiarissimo come responsabile di questa situazione, non limitata a Palermo ma diffusa in tutta Italia, sia la perdita della memoria storica: «Via, via, lontano da quella città che ha disprezzato probità e intelligenza, memoria, eredità di storia, arte, ha ucciso i deboli e i giusti. / Ma è Palermo o è Milano, Bologna, Brescia, Roma, Napoli, Firenze?» (Consolo, 1994, 125). Ne Lo Spasimo, questa situazione di crisi comporta una divaricazione tra letteratura e società. Chino ne matura la consapevolezza:

Un paradosso questo del mantello nero in cui si muta qui la toga di chi inquisisce e giudica usando la forza della legge. E per me anche letterario. Voglio dire: oltre che in Inghilterra, nella

[5] Da sottolineare come i quattro verbi (sparano-straziano-carbonizzano-spiaccicano) abbiano la stessa finale. 
Francia dello Stato e del Diritto è fiorita la figura del giustiziere che giudica e sentenzia fuori dalle leggi. Balzac, Dumas, Sue ne sono i padri, con filiazioni vaste, fino al Bernède e al Feuillade di Judex e al Natoli nostro, [...]. In questo Paese invece, in quest'accozzaglia di famiglie, questo materno confessionale d'assolvenza, dove lo stato è occupato da cosche o segrete sette di Dévorants, [...], dove tutti ci impegniamo, governanti e cittadini, ad eludere le leggi, a delinquere, il giudice che applica le leggi ci appare come un Judex, un giustiziere insopportabile, da escludere, da rimuovere. O da uccidere (Consolo, 1998, 129-130).

Il letterato, dunque, l'intellettuale vive in una condizione di esilio, non è adeguatamente apprezzato, tant'è che quando Chino tenta di fermare Borsellino mentre suona al citofono, il giudice si gira ma non lo riconosce. Nell'attentato viene colpito Erasmo che, morendo, recita due versi de $L a$ storia di la Baronissa di Carini, ad attestare come, anche se al presente la letteratura non è ascoltata, è da questa, voce della tradizione, della memoria storica, che deve venire la salvezza:

O gran mano di Diu, ca tantu pisi,

cala, manu di Diu, fatti palisi! (Consolo, 1998, 131). 


\section{Bibliografia}

Bouchard, Norma (2005). Vincenzo Consolo and the Postmodern Writing of Melancholy. Italica, 82, (1), 5 - 23.

Ciccarelli Andrea (a cura di) (2005). Intervista a Vincenzo Consolo. Italica, 82, (1), 92 - 97.

Consolo, Vincenzo (1988). Le pietre di Pantalica. Milano: Mondadori.

Consolo, Vincenzo (1992). Retablo. Milano: Mondadori.

Consolo, Vincenzo (1993). Fuga dall'Etna. Roma: Donzelli.

Consolo, Vincenzo (1994). L'olivo e l'olivastro. Milano: Mondadori.

Consolo, Vincenzo (1998). Lo Spasimo di Palermo. Milano: Mondadori.

Consolo, Vincenzo (1999). Di qua dal faro. Milano: Mondadori.

Consolo, Vincenzo (2006). «Ma la luna, la luna...». In Pintor Romera I. (a cura di). Lunaria. Vent'anni dopo. València: Generalitat Valenciana, Conselleria de Cultura, Educació i Esport.

Consolo, Vincenzo (2012). La mia isola è Las Vegas. Milano: Mondadori.

Donnarumma, Raffaele (2011). Storia, immaginario, letteratura: il terrorismo nella letteratura italiana (1969 - 2010). In Aa. Vv., Per Romano Luperini. Palermo: Palumbo.

Dotti, Ugo (2012). Il senso della storia nell'opera di Vincenzo Consolo. In Ugo Dotti, Gli scrittori e la storia. La narrativa dell'Italia unita e le trasformazioni del romanzo (da Verga a oggi). Torino: Nino Aragno editore.

Francese, Joseph (2015). Vincenzo Consolo: gli anni de «l'Unità» (1992 2012), ovvero la poetica della colpa - espiazione. Firenze: Firenze University Press.

Lollini, Massimo (2005). Intrecci mediterranei. La testimonianza di Vincenzo Consolo, moderno Odisseo. Italica, 82, (1), 24 - 43.

Luperini, Romano (1999). Controtempo. Napoli: Liguori.

Marcellesi - Giacomo, Mathée (2004). Vincenzo Consolo: l'alchimie du 
logos. Croniques italiennes. 2-3, 197 - 214.

Onofri, Massimo (2004). Il sospetto della realtà: Saggi e paesaggi italiani novecenteschi, Cava de' Tirreni: Avagliano.

O'Connell, Daragh (2004). Il dovere del racconto: Interview with Vincenzo Consolo. The Italianist. 24: II, 238 - 253. DOI: 10.1179 / ita.2004.24.2.238

O'Connell, Daragh (2008). Consolo narratore e scrittore palincestuoso. Quaderns d'Italià, (13), 161 - 184.

O'Connell, Daragh (2012). «Tizzone d'Inferno»: Sciascia on Joyce. Totomodo, 237 - 248.

Traina, Giuseppe (2001). Vincenzo Consolo. Fiesole: Cadmo.

\section{Summary}

Lo Spasimo di Palermo is example of a crisis that has repercussions on the expressive form: on the one hand, the consciousness that «sul ciglio dell'abisso la parola si raggela, si fa [...] simbolo sfuggente» comes out, on the other hand novel seems a "genere scaduto, corrotto, impraticabile». The generational conflict between Chino and Mauro is included in this sphere too. Hence it follows many solutions that lead to Consolo's «poema narrativo» and testify the prevailing disorientation: paratactic accumulation, metaphors, literary quotations, figures of speech. Pictorial suggestions find its centre in Raphael's Lo Spasimo di Sicilia, symbol of a pain that from Palermo arrives to Sicily and all world, while the spaces have a great importance and show the loss of historical memory, responsible for crisis.

Key words: Consolo, crisis, memory, history 
